NoTe

\title{
Association of Common LH Variant with Hyperfunctional Promoter in a Japanese Infertile Woman
}

\author{
SHUNYU LIU, TSUTOMU OGATA, TETSUO MARUYAMA*, YASUNORI YOSHIMURA* \\ AND BUNPEI ISHIZUKA** \\ Department of Endocrinology and Metabolism, National Research Institute for Child Health and Development, Tokyo 157-8535, \\ Japan \\ *Department of Obstetrics and Gynecology, Keio University School of Medicine, Tokyo 160-8582, Japan \\ **Department of Obstetrics and Gynecology, St. Marianna University School of Medicine, Kawasaki 216-0015, Japan
}

\begin{abstract}
A common LH variant (V-LH) with Trp8Arg and Ile15Thr is often associated with ovarian dysfunction primarily in the Japanese population, and the $L H B$ gene encoding V-LH is linked with a hyperfunctional promoter that could partly compensate for the somewhat weak biological effect of the V-LH in the Finnish and other several populations. We analyzed the promoter region in a Japanese infertile woman homozygous for the V-LH, to examine whether the hyperfunctional promoter is present or absent in the Japanese V-LH carriers with ovarian dysfunction. Direct sequencing was performed for a $661 \mathrm{bp}$ promoter region from -8 to $-668 \mathrm{bp}$ of $L H B$, revealing homozygosity for eight nucleotide substitutions $(-238 \mathrm{~A}>\mathrm{G},-276 \mathrm{G}>\mathrm{A},-489 \mathrm{C}>\mathrm{A},-490 \mathrm{~T}>\mathrm{A},-504 \mathrm{~T}>\mathrm{A},-506 \mathrm{~T}>\mathrm{C},-525 \mathrm{~T}>\mathrm{G}$, and $-552 \mathrm{C}>\mathrm{T})$ that are identical to those found in the hyperfunctional promoter. The results suggest that ovarian dysfunction frequently observed in the Japanese V-LH carriers would be due to some population-specific genetic and/or environmental factor(s) rather than to the lack of the hyperfunctional promoter and the resultant low biological effect of the V-LH. In addition, the tight linkage between the two missense substitutions in the coding region and the eight nucleotide substitutions in the promoter region of $L H B$ appears to be common to various ethnic groups.
\end{abstract}

Key words: LH variant, Trp8Arg and Ile15Thr, Promoter sequence, Ovarian dysfunction

(Endocrine Journal 52: 781-784, 2005)

A genetic variant of LH (V-LH) with two completely linked Trp8Arg and Ile15Thr substitutions in the LH $\beta$-subunit gene $(L H B)$ has been identified in various populations $[1,2]$. This common V-LH is immunologically undetectable when a monoclonal antibody recognizing an epitope present in the intact $\mathrm{LH} \alpha / \beta$ dimer is utilized. Trp8Arg is primarily responsible for the altered immunoreactivity, whereas Ile15Thr introduces an extra glycosylation site into the LH $\beta$ chain. The V-LH has a stronger in vitro bioactivity

Received: June 9, 2005

Accepted: August 17, 2005

Correspondence to: Dr. Tsutomu OGATA, Department of Endocrinology and Metabolism, National Research Institute for Child Health and Development, 2-10-1 Ohkura, Setagaya, Tokyo 1578535, Japan and a shorter circulatory half-life than the wildtype LH (WT-LH), and the overall hormonal function appears to be somewhat weaker in the V-LH than in the WT-LH $[1,2]$.

The functional difference between the V-LH and the WT-LH may predispose the V-LH carriers to ovarian disorders. Notably, association of the V-LH homozygosity with irregular menses and infertility, and that of the V-LH heterozygosity with infertility and premature ovarian failure (POF), have frequently been observed in the Japanese population [3-6], whereas such findings are absent in the Finnish population and have not clearly been demonstrated in other Caucasian populations $[1,2]$. In this regard, although $L H B$ encoding $\mathrm{V}$-LH is accompanied by additional eight nucleotide changes in the promoter region that enhance the promoter activity by $\sim 40 \%$ and compensate for the bio- 
logical effect of the relatively weak V-LH in the Finnish and other populations [7], the promoter region has not been analyzed in the Japanese population. Thus, if the $L H B$ encoding V-LH in the Japanese population is not accompanied by the hyperfunctional promoter, this may result in the reduced transcription of the variant $L H B$, increasing the susceptibility to the development of ovarian dysfunction in the Japanese VLH carriers. To examine this possibility, we analyzed the $L H B$ promoter sequences in a Japanese infertile woman homozygous for the V-LH.

\section{Case Report}

A 39-year-old Japanese woman was referred to St. Marianna University Hospital because of infertility and POF. Her menarche occurred at 12 years of age and was followed by irregular menses with $30-50$ day cycles. She married at 34 years of age, and visited a local hospital for irregular menses and infertility at 35 years of age. Repeated measurements of basal body temperature indicated no ovulation, and Fallopian obstruction and spermatogenic failure were excluded. Thus, she received artificial insemination with husband's semen for five times after ovulation induction with human menopausal gonadotropin (hMG) and human chorionic gonadotropin (hCG) treatment. However, this therapy was unsuccessful, and she had POF at 38 years of age.

Physical examination at our clinic revealed a normallooking woman with sufficient sexual development (breast, Tanner stage 5; pubic hair, Tanner stage 4). Her height was $153.0 \mathrm{~cm}(-0.9 \mathrm{SD})$, and her weight $55.2 \mathrm{~kg}(+0.3 \mathrm{SD})$. Routine laboratory tests were normal, and serum antinuclear antibodies and lupus anticoagulants were negative. Her karyotype was 46,XX in all the 50 lymphocyte examined. While hysteroscopy revealed normal uterus, laparoscopy showed hypoplastic ovaries and transvaginal ultrasonography failed to delineate follicles. Ovulation induction was unsuccessful, and she was placed on hormone replacement therapy from 40 years of age.

\section{Endocrine studies}

Serum LH and FSH were determined by DELFIA assay (Wallac, Turku, Finland) at the previous hospital at 35 years of age and by SPAC-S assay (Daiichi Radio- isotope Laboratory, Tokyo, Japan) at St. Marianna University Hospital at 39 years of age. Since the DELFIA assay uses two monoclonal antibodies both recognizing specific sites in the LH $\beta$ subunit, it detects both WT-LH and V-LH. By contrast, since the SPAC-S assay utilizes two monoclonal antibodies reacting with the LH $\beta$ subunit and intact $\alpha / \beta$ dimer, respectively, it detects WT-LH only. Serum prolactin was measured by SPAC-S assay, and estradiol and testosterone were obtained by radioimmunoassay at St. Marianna University Hospital.

The DELFIA assay showed that serum LH was $76 \mathrm{mIU} / \mathrm{mL}$ at baseline level (normal range at follicular phase, $2-8 \mathrm{mIU} / \mathrm{mL}$ ) and increased to a peak value of $372 \mathrm{mIU} / \mathrm{mL}$ during $\mathrm{GnRH}$ test $(100 \mu \mathrm{g}$ bolus i.v.; blood sampling at $0,30,60,90$, and $120 \mathrm{~min}$ ), and that serum FSH was $69 \mathrm{mIU} / \mathrm{mL}$ at baseline level (5-15 $\mathrm{mIU} / \mathrm{mL}$ ) and increased to a peak value of $165 \mathrm{mIU} /$ $\mathrm{mL}$ during GnRH test. The SPAC-S assay revealed that serum LH was undetectable $(<0.2 \mathrm{mIU} / \mathrm{mL})$ and FSH $83 \mathrm{mIU} / \mathrm{mL}$. The undetectable LH value was confirmed by three time measurements. Serum prolactin was $12 \mathrm{ng} / \mathrm{mL}(1-15 \mathrm{ng} / \mathrm{mL})$, estradiol $12 \mathrm{pg} / \mathrm{mL}(20$ $200 \mathrm{pg} / \mathrm{mL})$, and testosterone $0.5 \mathrm{ng} / \mathrm{mL}(0.1-0.6 \mathrm{ng} /$ $\mathrm{mL}$ ). An hMG or hCG test was not carried out.

\section{Molecular studies}

This study has been approved by the Institutional Review Board Committee at National Center for Child Health and Development. Leukocyte genomic DNA of this patient was amplified for the coding region and the $661 \mathrm{bp}$ promoter region from -8 to $-668 \mathrm{bp}$ of $L H B$ by polymerase chain reaction (PCR) with the primers described previously [3,7]. Subsequently, the PCR products were subjected to direct sequencing from both directions on a CEQ 8000 autosequencer (Beckman Coulter, Fullerton, CA).

Direct sequencing revealed Trp8Arg $(22 \mathrm{~T}>\mathrm{C})$ and Ile15Thr $(44 \mathrm{~T}>\mathrm{C})$ in the coding region (Fig. 1-A). Furthermore, the following eight nucleotide changes were found in the promoter region: $-238 \mathrm{~A}>\mathrm{G},-276 \mathrm{G}$ $>\mathrm{A},-{ }_{489} \mathrm{C}>\mathrm{A},-490 \mathrm{~T}>\mathrm{A},-504 \mathrm{~T}>\mathrm{A},-506 \mathrm{~T}>\mathrm{C},-525 \mathrm{~T}$ $>\mathrm{G}$, and $-552 \mathrm{C}>\mathrm{T}$ (Fig. 1-B). All the substitutions were present in a homozygous condition. 
(A)

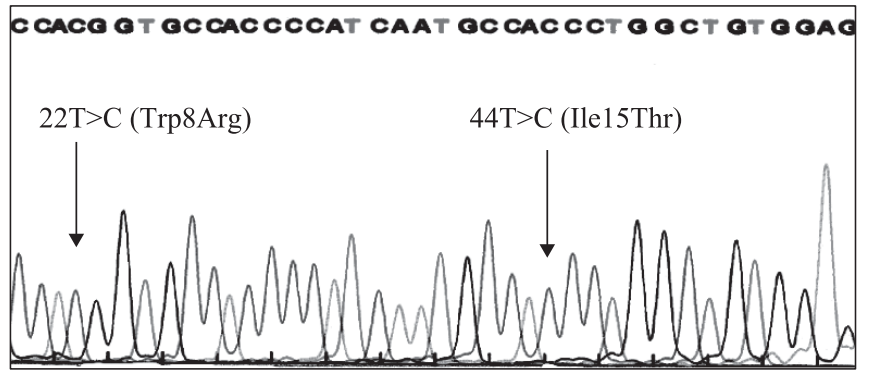

(B)

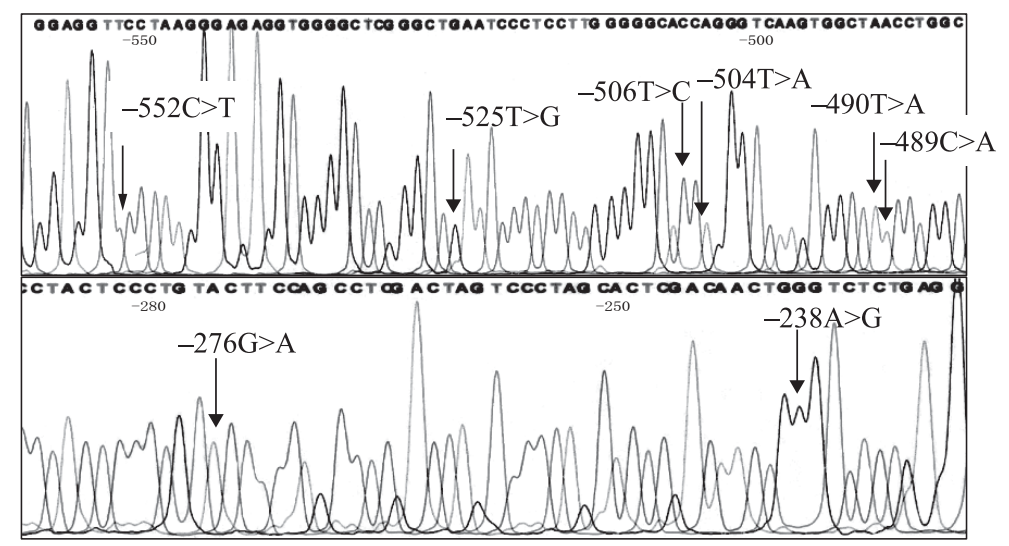

Fig. 1. A. An electrochromatogram of the coding region showing homozygosity for $22 \mathrm{~T}>\mathrm{C}$ ( $\operatorname{Trp} 8 \mathrm{Arg}$ ) and $44 \mathrm{~T}>\mathrm{C}$ (Ile15The). B. An electrochromatogram of the promoter region showing homozygosity for $-238 \mathrm{~A}>\mathrm{G},-276 \mathrm{G}>\mathrm{A},-489 \mathrm{C}>\mathrm{A},-490 \mathrm{~T}>\mathrm{A}$, $-504 \mathrm{~T}>\mathrm{A},-506 \mathrm{~T}>\mathrm{C},-525 \mathrm{~T}>\mathrm{G}$, and $-552 \mathrm{C}>\mathrm{T}$.

\section{Discussion}

This infertile woman was homozygous for the V-LH with Trp8Arg and Ile15Thr substitutions. Consistent with this, her LH was detected by the DELFIA assay and undetected by the SPAC-S assay. The results provide further support for the previous finding that the $\mathrm{V}-\mathrm{LH}$ is frequently associated with ovarian dysfunction in the Japanese population [3-6].

The promoter region analysis showed eight nucleotide substitutions. Since they are identical to those found in the hyperfunctional promoter of the Finnish and other several populations [7], the results would argue against the possibility that ovarian dysfunction frequently observed in the Japanese V-LH carriers may be ascribed to the lack of the hyperfunctional promoter and the resultant reduction in transcription of the $L H B$ encoding V-LH. It is inferred, therefore, that some population-specific genetic and/or environmental factor(s) is involved in the development of ovarian dysfunction in the Japanese V-LH carriers.

Although the underlying genetic and/or environmental factor(s) remains to be clarified, a factor(s) common to the Japanese population, which is absent from the $L H B$ encoding the V-LH, may be relevant to the development of ovarian dysfunction. This notion assumes that an interaction between such a factor(s) and the V-LH with somewhat lower biological function $[1,2]$ exerts an additive or synergic deleterious effect, leading to the occurrence of ovarian dysfunction in the Japanese V-LH carriers. In this regard, since the prevalence of POF has been reported to be lower in the Japanese population than in the Caucasian populations [8], this may argue against this possibility. However, such epidemiological data remain poor, and even a factor(s) common to the Japanese could specifically influence the V-LH carriers. For example, there might be an LH receptor variant that is widespread in the Japanese population and is associated with a lower affinity to the V-LH but not to the WT-LH. Furthermore, since such a factor(s) should also be present in males as well as in females, this would be consistent with the development of testicular dysfunction in a Japanese male patient homozygous for the V-LH [9].

By contrast, it appears unlikely that such a factor(s) resides in a region tightly linked to the $L H B$ encoding 
V-LH. Since an additional substitution has not been identified in the coding sequences of the $L H B$ encoding V-LH in the Japanese as well as in other populations [1-6], this would exclude the possibility that the $\mathrm{V}$-LH protein in the Japanese population is accompanied by a specific structural alteration affecting the biological function such as the binding capacity to the receptor. Similarly, since serum V-LH value determined by the DELFIA assay was markedly elevated, this would argue against the possibility that the transcription of the $L H B$ encoding the V-LH is reduced in the Japanese V-LH carriers, because of the presence of a hidden hypofunctional polymorphism in the noncoding region such as a more distal promoter sequence and an intronic sequence.

Furthermore, the results imply that the linkage between the two missense substitutions in the coding region and the eight nucleotide substitutions in the promoter region may be common to various ethnic groups. Although it remains to be clarified why such a linkage could be conserved in different populations, it is possible that the combination of the Trp8Arg and Ile15Thr substitutions and the hyperfunctional promoter may have permitted the preservation of grossly normal biological effect of the hormone, thereby protecting the V-LH from the evolutional selection $[1,2$, 7]. In this regard, the V-LH may be gradually eliminated from the Japanese population, because of the possible lower biological function. This notion awaits further analysis of the LHB gene encoding the V-LH in the Japanese and other populations.

\section{Acknowledgements}

This study was supported by a grant for Child Health and Development from the Ministry of Health, Labor, and Welfare (17C-2) and by a Grant-in-Aid for Scientific Research on Priority Areas (16086215).

\section{References}

1. Themmen APN, Huhtaniemi IT (2000) Mutations of gonadotropins and gonadotropin receptors: elucidating the physiology and pathophysiology of pituitarygonadal function. Endocr Rev 21: 551-583.

2. Lamminen T, Huhtaniemi I (2001) A common genetic variant of luteinizing hormone; relation to normal and aberrant pituitary-gonadal function. Eur J Pharmacol 414: $1-7$.

3. Furui K, Suganuma N, Tsukahara S, Asada Y, Kikkawa F, Tanaka M, Ozawa T, Tomoda Y (1994) Identification of two point mutations in the gene coding luteinizing hormone ( $\mathrm{LH}$ ) beta-subunit, associated with immunologically anomalous LH variants. J Clin Endocrinol Metab 78: 107-113.

4. Suganuma N, Furui K, Furuhashi M, Asada Y, Kikkawa F, Tomoda Y (1995) Screening of the mutations in luteinizing hormone beta-subunit in patients with menstrual disorders. Fertil Steril 63: 989-995.

5. Takahashi K, Kurioka H, Ozaki T, Kanasaki H, Kohsaka M, Miyazaki K, Karino K (1998) Increased prevalence of luteinizing hormone beta-subunit variant in Japanese infertility patients. Hum Reprod 13: 3338-3344.

6. Takahashi K, Kurioka H, Ozaki T, Kanasaki H, Kohsaka M, Miyazaki K, Karino K (1998) Increased prevalence of luteinizing hormone beta-subunit variant in patients with premature ovarian failure. Fertil Steril 71: 96101.

7. Jiang $\mathrm{M}$, Pakarinen $\mathrm{P}$, Zhang FP, El-Hefnawy $\mathrm{T}$, Koskimies P, Pettersson K, Huhtaniemi I (1999) A common polymorphic allele of the human luteinizing hormone beta-subunit gene: additional mutations and differential function of the promoter sequence. Hum Mol Genet 8: 2037-2046.

8. Luborsky JL, Meyer P, Sowers MF, Gold EB, Santoro N (2002) Premature menopause in a multi-ethnic population study of the menopause transition. Hum Reprod 18: 199-206.

9. Shiraishi K, Naito K (2003) Fertile eunuch syndrome with the mutations (Trp8Arg and Ile15Thr) in the beta subunit of luteinizing hormone. Endocr J 50: 733-737. 